\title{
A análise seminal deve ser requisitada para homens com histórico de fertilidade prévia?
}

\author{
Should semen analysis be requested for men with a history of previous fertility? \\ Eleonora Bedin Pasqualotto ${ }^{1}$, Ramon Venzon Ferreira ${ }^{2}$, Gabriela Poglia Fonseca ${ }^{2}$, Bibiana Eliza Zago ${ }^{2}$, \\ Cláudio Garbin Júnior ${ }^{2}$, Fábio Firmbach Pasqualotto ${ }^{3}$
}

\begin{abstract}
Resumo
Objetivos: determinar se a história prévia de fertilidade pode predizer o atual status de fertilidade de um paciente masculino examinado por infertilidade do casal. Métodos: estudo retrospectivo envolvendo análises seminais de 183 pacientes consecutivos subférteis avaliados entre setembro de 2002 e março de 2004 . Foram excluídos do estudo os pacientes que haviam se submetido a radioterapia, quimioterapia, orquiectomia ou vasectomia. Os valores médios de todas as análises foram usados em pacientes com múltiplas análises de sêmen. Pacientes com concentração espermática superior a $20 \times 10^{6}$ espermatozóides/mL, motilidade superior a 50\% e espermatozóides com morfologia estrita superior a 14\% foram considerados normais. Os pacientes foram divididos em dois grupos, segundo o status de fertilidade: infertilidade primária (118 pacientes) e infertilidade secundária (65 pacientes). Os dados foram analisados pelos testes estatísticos $\chi^{2}$ e teste $t$ de Student. Resultados: não houve diferença na idade média entre os pacientes com infertilidade primária, 37,3 $\pm 6,3$, e

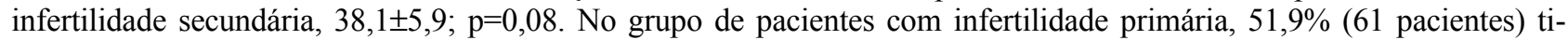
veram uma concentração espermática normal, 70,3\% (83 pacientes) tiveram a motilidade espermática normal e 26,37\% (31 pacientes), por sua vez, morfologia normal. No grupo de pacientes com infertilidade secundária, 53,8\% (35 pacientes) tiveram concentração espermática normal, 75,4\% (49 pacientes) tiveram motilidade espermática normal e 32,3\% (21 pacientes), morfologia normal. Nenhuma diferença significativa foi detectada na concentração espermática $\left(21,3 \times 10^{6} / \mathrm{mL}\right.$ versus $\left.23,1 \times 10^{6} / \mathrm{mL} ; \mathrm{p}=0,07\right)$, motilidade $(45,2$ versus $48,1 \% ; \mathrm{p}=0,08)$ e morfologia $(6,1$ versus $6,4 \% ; \mathrm{p}=0,09)$ entre os grupos de pacientes com infertilidade primária e secundária. Conclusões: a análise seminal deve ser solicitada mesmo em casos de fertilidade masculina prévia. Os médicos não devem presumir que um paciente possui uma análise seminal normal, baseados no fato de este possuir história de estabelecimento de uma gravidez no passado.
\end{abstract}

PALAVRA-CHAVE: Infertilidade masculina; Semen/análise; Fertilidade; Contagem de espermatozóides

\section{Abstract}

Purpose: to determine if the previous fertility history can predict current fertility status of a patient examined for couple's infertility. Methods: retrospective study involving semen analyses from 183 consecutive subfertile patients evaluated from September 2002 to March 2004. We excluded those patients who had undergone radio or chemotherapy, orchiectomy or vasectomy. Mean values of all analyses were used for patients with multiple semen analysis. Patients with more than $20 \times 10^{6}$ sperm $/ \mathrm{mL}$, motility higher than $50 \%$ and with normal strict sperm morphology higher than $14 \%$ were considered normal. Patients were divided into two groups, according to the fertility status: primary infertility (118 patients) and secondary infertility (65 patients). Data were analyzed according to the $\chi^{2}$ test and the Student $t$-test. Results: no differences were detected in the mean age between patients with primary infertility, $37.3 \pm 6.3$, and secondary infertility, $38.1 \pm 5.9 ; \mathrm{p}=0.08$. In the group of patients with primary infertility, $51.9 \%$ (61 patients) had a normal sperm concentration, $70.3 \%$ ( 83 patients) had normal sperm motility and $26.3 \%$ (31 patients) had normal sperm morphology. In the group of patients with secondary infertility, 53.8\% (35 patients) had normal sperm concentration, 75.4\% (49 patients) had normal sperm motility and 32.3\%

Centro de Ciências Biológicas e da Saúde da Universidade de Caxias do Sul - UCS - Caxias do Sul (RS) e Conception - Centro de Reprodução Humana - Caxias do Sul (RS) - Brasil.

1 Professora Titular de Ginecologia da Faculdade de Medicina da Universidade de Caxias do Sul - UCS - Caxias do Sul (RS); Diretora Geral do Centro de Reprodução Humana - Caxias do Sul (RS) - Brasil.

2 Acadêmicos de de Medicina da Universidade de Caxias do Sul - UCS - Caxias do Sul (RS) - Brasil.

3 Professor Titular de Anatomia e Embriologia da Faculdade de Medicina da Universidade de Caxias do Sul - UCS - Caxias do Sul (RS); Diretor do Centro de Reprodução Humana - Caxias do Sul (RS) - Brasil.

Correspondência: Fabio Firmbach Pasqualotto

Rua Pinheiro Machado, 2569, sl 23/24 - Bairro São Pelegrino - 95020-172 - Caxias do Sul - RS - Telefone: (54) 3214-4095 - Fax: (54) 3215-1695 e-mail: fabio@conception-rs.com.br

Recebido em: 16/5/2006 
(21 patients) had normal sperm morphology. No significant differences were detected in sperm concentration $\left(21.3 \times 10^{6} / \mathrm{mL}\right.$ versus $\left.23.1 \times 10^{6} / \mathrm{mL} ; \mathrm{p}=0.07\right)$, motility (45.2 versus $48.1 \%$; $\left.\mathrm{p}=0.08\right)$ and morphology $(6.1$ versus $6.4 \%$; $\mathrm{p}=0.09)$ between groups of patients with primary and secondary infertility. Conclusions: semen analysis should be requested even in cases of prior male fertility. Physicians should not presume a patient to have a normal semen analysis based on his previous history of initiating a pregnancy.

KEYWORDS: Infertility, male; Semen/analysis; Fertility; Sperm count

\section{Introdução}

A avaliação da qualidade seminal constitui um dos primeiros aspectos a ser avaliado na investigação de infertilidade dos casais, sendo recomendada mesmo em trabalhos que sugerem baixo poder diagnóstico dos valores de referência dos parâmetros seminais ${ }^{1,2}$. Embora a análise seminal (AS) não seja, isoladamente, considerada fundamental para o diagnóstico de infertilidade masculina ${ }^{3}$, ela assume importante papel na avaliação de pacientes com história prévia de gravidez e que, no presente momento, apresentam queixas de infertilidade secundária. Muitos pacientes e até mesmo colegas presumem, erroneamente, que um homem, por já ter estabelecido uma gravidez prévia, tenha seu status reprodutivo firmado.

Por outro lado, a análise seminal básica tem sido utilizada para avaliar os parceiros de casais com queixas de infertilidade ${ }^{4}$. Apesar de as técnicas convencionais para avaliação seminal terem sido padronizadas pela Organização Mundial da Saúde (OMS), muitas dúvidas ainda persistem sobre o real valor dos resultados bem como sobre os valores de normalidade propagados pela $\mathrm{OMS}^{4-7}$. Estudo demonstra que até $87 \%$ de uma população de homens comprovadamente férteis e desejosos em realizar uma cirurgia de vasectomia apresentam valores de morfologia espermática inferiores aos da OMS ${ }^{4}$.

Contudo, na literatura, há relatos consistentes sobre a piora na qualidade seminal de forma progressiva em pacientes com varicocele ${ }^{2,8}$, aumento na idade ${ }^{9}$, exposição ao fumo ${ }^{10}$ e outras entidades associadas à piora na qualidade seminal, guardando proporcionalidade com o tempo de exposição. A varicocele é um grande exemplo de que uma patologia pode alterar a qualidade seminal com o passar dos anos. Estudos demonstram que ela acomete de 15 a $20 \%$ da população masculina em geral. Por outro lado, 30 a $40 \%$ dos homens com infertilidade primária apresentam varicocele $\mathrm{e}$ até $80 \%$ dos homens com infertilidade secundária apresentam varicocele clinicamente detectável, de- monstrando que ela pode causar danos testiculares com o passar dos anos ${ }^{11}$.

Com relação ao fator idade, estudos demonstram que a qualidade seminal piora após os 40 anos de idade. Estas alterações podem variar desde diminuição na concentração de espermatozóides até diminuição na motilidade, parâmetros de cinética da movimentação até piora na morfologia espermática $^{12,13}$.

O uso de cigarros, com o passar dos anos, também pode causar piora na qualidade do ejaculado. Estudos demonstram que ele pode causar danos, como a diminuição no volume seminal até a fragmentação no DNA dos espermatozóides ${ }^{14,15}$. Além do cigarro, uma série de substâncias de exposição ocupacional têm sido consideradas deletérias para o sistema reprodutivo masculino, como pesticidas e agrotóxicos ${ }^{16,17}$.

O objetivo do nosso estudo foi avaliar a importância da realização da análise seminal em pacientes com infertilidade secundária, uma vez que há a idéia de que a fertilidade prévia pudesse ser um indicador do status seminal atual.

\section{Métodos}

Este estudo retrospectivo foi aprovado pelo Comitê de Ética e Pesquisa da Universidade de Caxias do Sul (UCS) e todos os participantes do estudo assinaram um termo de consentimento informado. O presente estudo foi realizado de setembro de 2002 a março de 2004, quando foram analisadas 183 amostras seminais consecutivas de pacientes subférteis investigados por infertilidade.

Os pacientes foram encaminhados para investigação de infertilidade por ginecologistas, urologistas ou procura espontânea. A avaliação dos pacientes foi realizada por um urologista especialista em infertilidade masculina (FFP). Foram excluídos do estudo os pacientes que haviam se submetido a radioterapia, quimioterapia, orquiectomia, vasectomia ou qualquer outro tipo de cirurgia urológica, porque, sabidamente, estes pacientes provavelmente teriam alterações seminais. 
Após assinarem o consentimento livre e esclarecido, os pacientes foram orientados a manter um periodo de abstinência de 48 a 72 horas. Todos os pacientes foram orientados a coletar suas amostras seminais, por masturbação, dentro de um recipiente plástico estéril. As amostras foram submetidas a liquefação a $37^{\circ} \mathrm{C}$ por 30 minutos, sendo avaliados os seguintes parâmetros seminais: volume seminal, percentual de motilidade espermática e concentração total de espermatozóides móveis. O volume do ejaculado foi medido por aspiração de toda a amostra com o auxílio de uma pipeta graduada acoplada a um pipetador eletrônico. Após misturar cuidadosamente a amostra seminal por completo, com o auxílio de um aparelho misturador de amostras, as mesmas foram avaliadas manualmente pelo pesquisador. Para cada mensuração, uma alíquota liquefeita de $5 \mu \mathrm{L}$ foi inserida em uma câmara de contagem Microcell $^{\circledR}$, descartável, com $20 \mu \mathrm{m}$ de profundidade até seu preenchimento integral, com o auxílio de uma pipeta de pressão positiva para determinação da concentração e motilidade espermáticas. Estas foram analisadas manualmente, com o uso de um microscópio óptico equipado com uma objetiva de contraste de fase de 10X e uma magnificação de 200X.

Dois esfregaços de cada amostra foram preparados em lâminas de microscopia para serem fixados e corados. O método utilizado para fixação depende da técnica de coloração a ser aplicada. Para a realização do presente estudo, utilizou-se apenas um método de coloração, o Diff-Quick (Baxter Healthcare Corporation, Inc.). Após a lâmina ter secado ao ar ambiente, a mesma foi identificada com o nome do paciente, número de acesso e data. Esta lâmina, então, foi imersa na solução fixadora de Diff-Quick $(1,8 \mathrm{mg} / \mathrm{L}$ de corante triarylmetano, $100 \%$ PDC [corante puro] em álcool metílico) cinco vezes, por um segundo a cada vez, com um intervalo de um segundo entre cada imersão. Após aproximadamente 15 minutos, a lâmina já estava seca, para dar início à próxima etapa da coloração, e ser imersa na solução I de Diff-Quick (1 g/L de corante xanthene 100\% PDC, tampão e azido de sódio $(0,01 \%))$ por três vezes, por um segundo a cada vez, com intervalo de um segundo. O excesso do corante foi retirado e, imediatamente, a lâmina imersa na solução II de Diff-Quick (1,25 g/L corante de tiazina, 100\% PDC $(0,625 \mathrm{~g} / \mathrm{L}$ azure A e $0,625 \mathrm{~g} / \mathrm{L}$ azul de metileno)) por cinco vezes, por um segundo a cada vez, com intervalo de um segundo. A lâmina foi lavada em água deionizada para remover qualquer excesso de corante; deixou-se a lâmina corada em local apropriado para secar (suporte de lâminas).
No estudo aqui apresentado, foram avaliados um mínimo de 200 espermatozóides corados por paciente. Para avaliar a morfologia, a leitura foi realizada por meio de uma objetiva de imersão com óleo antidissipador de fluorescência, com magnificação de 100X. Apenas espermatozóides totalmente desenvolvidos foram reconhecidos e classificados.

Os pacientes que apresentaram uma concentração espermática superior a 20 milhões de espermatozóides $/ \mathrm{mL}$ ou motilidade superior a $50 \%$ (critérios de normalidade da OMS) e morfologia estrita dos espermatozóides superior a $14 \%$ foram considerados normais ${ }^{18,19}$.

Os prontuários dos pacientes foram avaliados a fim de determinar se estes possuíam ou não uma história de fertilidade prévia. Após esta revisão, os pacientes foram divididos em dois grupos, segundo o padrão de infertilidade atual: com infertilidade primária (118 pacientes) e com infertilidade secundária (65 pacientes). Foram quantificados, em ambos os grupos, pacientes com parâmetros seminais normais. Posteriormente, foram comparadas as médias de idade e parâmetros seminais entre os pacientes com infertilidade primária e com infertilidade secundária.

Os dados foram analisados pelos recursos estatísticos $\mathrm{x}^{2} \mathrm{e}$ teste $t$ de Student. O nível de diferença estatística estabelecido foi $5 \%$ (valor de $p<0,05$ foi considerado estatisticamente significativo). A informação foi analisada pelo programa Statistical Package for Social Sciences, SPPS, versão 11.0 (SPSS Inc., Chicago, IL, EUA).

\section{Resultados}

A idade média no grupo com infertilidade primária foi de $37,3 \pm 6,3$ e no grupo com infertilidade secundária de $38,1 \pm 5,9(\mathrm{p}=0,08)$. No grupo com infertilidade primária, $51,9 \%$ (61 pacientes) apresentaram uma concentração espermática normal, $70,3 \%$ (83 pacientes) tiveram a motilidade espermática normal e 26,3\% (31 pacientes), por sua vez, morfologia normal. No grupo de pacientes com infertilidade secundária, 53,8\% (35 pacientes) apresentaram a concentração espermática normal, 75,4\% (49 pacientes) tiveram a motilidade espermática normal e 32,3\% (21 pacientes), por sua vez, morfologia normal (Tabela 1). Nenhuma diferença significativa foi detectada na concentração espermática $\left(21,3 \times 10^{6} / \mathrm{mL}\right.$ versus $\left.23,1 \times 10^{6} / \mathrm{mL} ; \mathrm{p}=0,07\right)$, motilidade $(45,2$ versus $48,1 \% ; p=0,08)$ e morfologia $(6,1$ versus $6,4 \% ; p=0,09)$ respectivamente entre os grupos avaliados (Tabela 2). 
Assim sendo, não foram encontradas quaisquer diferenças estatísticas na idade e nos parâmetros seminais analisados entre os grupos de infertilidade primária e de infertilidade secundária.

\section{Discussão}

A análise seminal é amplamente utilizada na avaliação do fator masculino da fertilidade ${ }^{3,9,10}$. Entretanto, a literatura atual ainda é escassa no que tange à comparação entre a análise seminal e a história de fertilidade anterior como fatores definidores do atual status seminal. Nossos resultados mostram que a história de fertilidade prévia não pode ser usada de maneira única e exclusiva como fator preditivo do potencial fértil dos pacientes masculinos com infertilidade secundária. Esta informação é de grande valia, uma vez que reforça a necessidade da análise seminal na investigação da infertilidade secundária. Em um cenário secundário, estes resultados possuem repercussões econômicas importantes, principalmente em países onde tanto os pacientes como os sistemas de saúde apresentam dificuldades econômicas, já que a análise seminal se faz necessária quase que na totalidade dos casos de infertilidade secundária.

Este estudo demonstrou que não existe diferença estatisticamente significativa entre as amostras seminais provenientes de um paciente com infertilidade primária comparadas àquelas provenientes de pacientes com infertilidade secundária. Dentre os parâmetros avaliados na análise seminal, o critério que mais diferiu dos critérios de normalidade foi o da morfologia espermática. Apenas pouco mais de $30 \%$ dos pacientes com infertilidade secundária apresentaram critérios considerados normais, segundo
Tygerberg, para morfologia espermática ${ }^{19}$. Interessantemente, a morfologia espermática é o critério considerado o mais importante para que o paciente consiga estabelecer gravidez ${ }^{3}$. Por outro lado, este estudo demonstrou que aproximadamente metade dos pacientes avaliados apresenta concentração espermática normal e até $25 \%$ dos pacientes apresentam motilidade considerada normal, segundo os critérios de normalidade da $\mathrm{OMS}^{18}$. Estes dados demonstram que, em uma grande porcentagem de pacientes, a morfologia espermática está alterada se avaliada de acordo com os critérios estritos de Tygerberg ${ }^{19}$.

Porém, o dado mais importante avaliado neste estudo foi que não existem diferenças nos parâmetros avaliados, quando comparados pacientes com infertilidade primária e secundária. Deste modo, há grande tendência à conclusão de que o paciente com infertilidade secundária, o qual já engravidou sua esposa e agora está apresentando dificuldades para conseguir estabelecer gravidez novamente, tenha apresentado piora na qualidade seminal com o passar dos anos. Esta ligação entre a variável tempo e o declínio da qualidade seminal, levando à infertilidade secundária, pode ser modulada de maneira evidente por diferentes fatores, entre eles a varicocele ${ }^{2,8,11}$, fumo ${ }^{10,14}$ e a idade ${ }^{9,12,13}$, entre outros. O objetivo do nosso estudo não foi avaliar as causas da infertilidade secundária e comparar com a análise seminal, mas apenas responder à pergunta, muitas vezes realizada no consultório médico, de que se o homem que possui um filho gerado com seu próprio material genético deve realizar um espermograma caso não consiga que sua parceira engravide novamente. Por outro lado, é importante destacar algumas causas de possivel piora na qualidade seminal que podem ser as responsáveis pelo quadro de infertilidade secundária.

Tabela 1 - Resultados dos pacientes com análise seminal normal: infertilidade primária e infertilidade secundária.

\begin{tabular}{lcc}
\hline Variáveis & Infertilidade primária (n/\%) & Infertilidade secundária (n/\%) \\
\hline Concentração espermática normal $\left(>20 \times 10^{6}\right.$ espermatozóides $\left./ \mathrm{mL}\right)$ & $61 / 51,9 \%$ & $35 / 53,8 \%$ \\
Motilidade espermática normal $(>50 \%)$ & $83 / 70,33 \%$ & $49 / 75,38 \%$ \\
Morfologia espermática normal $(>14 \%)$ & $31 / 26,27 \%$ & $21 / 32,3 \%$ \\
\hline
\end{tabular}

Tabela 2 - Análise da idade e parâmetros seminais: infertilidade primária e infertilidade secundária.

\begin{tabular}{lccc}
\hline Variáveis & $\begin{array}{c}\text { Infertilidade primária } \\
\text { (média } \pm \text { desvio padrão) }\end{array}$ & $\begin{array}{c}\text { Infertilidade secundária } \\
\text { (média } \pm \text { desvio padrão) }\end{array}$ & Valor de $\mathbf{p}$ \\
\hline Idade (anos) & $37,3 \pm 6,3$ & $38,1 \pm 5,9$ & 0,08 \\
Concentração espermática & $21,3 \times 10^{6}$ & $23,1 \times 10^{6}$ & 0,07 \\
(espermatozóides/mL) & & & 0,08 \\
Motilidade espermática & $45,2 \%$ & $48,1 \%$ & 0,09 \\
Morfologia espermática & $6,1 \%$ & $6,4 \%$ & \\
\hline
\end{tabular}

Valor de $p<0,05$ foi considerado estatisticamente significativo. 
A varicocele é a causa passivel de correção mais freqüente nos homens que buscam avaliação em clínicas de infertilidade. Existem evidências de que a varicocele pode ter um efeito deletério progressivo nos testículos, levando à associação de perda de função reprodutiva ao longo do tempo se não for corrigida ${ }^{2}$. Estes dados, somados à prevalência da varicocele na população em geral $(15 \%)$ e nos homens com infertilidade secundária (que varia de 69 a $84 \%$, com uma média de $80 \%)^{2,8,20,21}$, sugerem uma importante interação entre a varicocele como principal causa de infertilidade secundária ${ }^{8,20,21}$ e alteração do perfil reprodutivo masculino ao longo dos anos.

Os efeitos deletérios da mesma parecem ser progressivos com a idade, visto que a varicocele pode estar presente já na adolescência ${ }^{8,20,21}$. Estudo anterior, corroborando nossos achados, demonstram que determinado individuo, mesmo com uma análise seminal prévia normal ou história de fertilidade prévia, está sob risco de subseqüente perda da função testicular e infertilidade ${ }^{2}$.

Outra condição que estabelece um declínio na capacidade reprodutiva masculina de maneira progressiva é o tabagismo. Embora esta afirmação seja controversa na literatura atual ${ }^{16,17}$, existem inúmeros trabalhos demonstrando este efeito deletério do cigarro sobre a fertilidade masculina ${ }^{14,15}$. Uma meta-análise demonstra que pacientes fumantes apresentam um decréscimo médio de $10 \%$ na motilidade espermática, $13 \%$ na concentração espermática e $3 \%$ na morfologia espermática ${ }^{22}$. O volume seminal apresenta-se diminuído em pacientes fumantes, estratificados de acordo com o número de cigarros dia, quando comparados aos pacientes não fumantes ${ }^{14,23}$. O tabagismo pode igualmente causar diminuição da fertilidade por alterar os níveis hormonais séricos de testosterona e estradiol bem como alteração no DNA dos espermatozóides ${ }^{14,15}$. A fertilidade masculina fica prejudicada pelo tabagismo, na medida em que ocorre um decréscimo nas taxas de gravidez e alteração nos parâmetros seminais de pacientes tabagistas ${ }^{23}$. Por isso, é proposto que homens com qualidade seminal limitrofe, os quais pretendam ter filhos, talvez sejam beneficiados com o abandono do cigarro. Contudo, esta medida possui dados limitados na tentativa de recuperar a função espermática ${ }^{24}$.

O envelhecimento acarreta alterações sistêmicas no organismo. Neste contexto, incluem-se modificações morfo-fisiológicas testiculares e, conseqüentemente, na função reprodutiva masculina ${ }^{9,12,13,24,25}$. Homens mais velhos apresentam menor volume seminal, concentração total de espermatozóides, vitalidade e aumento na morfologia espermática anormal com diminuição das formas normais e au- mento na quantidade de espermatozóides com gotas citoplasmáticas $^{9,12}$. Em recente estudo que avaliou parâmetros seminais e hormonais em homens de acordo com a idade, foi observada uma tendência à diminuição da concentração espermática e da motilidade espermática normal. Os níveis do hormônio foliculo-estimulante (FSH) tendem a aumentar a longo do tempo e a morfologia espermática tende a decrescer a partir dos 45 anos de idade ${ }^{9}$.

Por estes fatores, alguns modificáveis (varicocele e tabagismo) e outros não (envelhecimento), fica evidente que os individuos masculinos ao longo de suas vidas são expostos a diferentes agentes que podem modificar seu status reprodutivo. Desta maneira, ao contrário do que acontece na mulher, a história reprodutiva masculina prévia não apresenta critérios definitivos para sua capacidade reprodutiva atual ${ }^{2}$.

Em conclusão, a análise seminal é a base na avaliação da infertilidade masculina, mesmo em casos de fertilidade masculina previamente provada. Os médicos não devem presumir que um paciente possua uma análise seminal normal, baseados no fato de este apresentar história de estabelecimento de uma gravidez no passado.

\section{Referências}

1. Álvarez C, Castilla J, Martínez L, Ramírez JP, Vergara F, Gaforio JJ. Biological variation of seminal parameters in healthy subjects. Hum Reprod. 2003; 18(10):2082-8.

2. Cozzolino DJ, Lipshultz LI. Varicocele as a progressive lesion: positive effect of varicocele repair. Hum Reprod Update. 2001;7(1):55-8.

3. Guzick DS, Overstreet JW, Factor-Litvak P, Brazil CK, Nakajima ST, Coutifaris C, et al. Sperm morphology, motility, and concentration in fertile and infertile men. N Engl J Med. 2001;345(19):1388-93.

4. Pasqualotto FF, Sobreiro BP, Hallak J, Athayde KS, Pasqualotto EB, Lucon AM. High percentage of abnormal semen parameters in a prevasectomy population. Fertil Steril. 2006;85(4):954-60.

5. Menkveld R, Wong WY, Lombard CJ, Wetzels AM, Thomas CM, Merkus HM, Steegers-Theunissen RP. Semen parameters, including WHO and strict criteria morphology, in a fertile and subfertile population: an effort towards standardization of in-vivo thresholds. Hum Reprod. 2001;16(6):1165-71.

6. Nallella KP, Sharma RK, Aziz N, Agarwal A. Significance of sperm characteristics in the evaluation of male infertility. Fertil Steril. 2006;85(3):629-34.

7. van der Merke FH, Kruger TF, Oehnnger SC, Lombard $\mathrm{CJ}$. The use of semen parameters to identify the 
subfertile male in the general population. Gynecol Obstet Invest. 2005;59(2):86-91.

8. Jarow JP. Effects of varicocele on male fertility. Hum Reprod Update. 2001;7(1):59-64.

9. Pasqualotto FF, Sobreiro BP, Hallak J, Pasqualotto EB, Lucon AM. Sperm concentration and normal sperm morphology decrease and follicle-stimulating hormone level increases with age. BJU Int. 2005;96(7):1087-91.

10.Trummer H, Habermann H, Haas J, Pummer $\mathrm{K}$. The impact of cigarette smoking on human semen parameters and hormones. Hum Reprod. 2002;17(6):1554-9.

11.Pasqualotto FF, Lucon AM, de Goes PM, Sobreiro BP, Hallak J, Pasqualotto EB, et al. Semen profile, testicular volume and hormonal levels in infertile patients with varicoceles compared with fertile men with and without varicoceles. Fertil Steril. 2005;83(1):74-7

12.Kidd SA, Eskenazi B, Wyrobek AJ. Effects of male age on semen quality and fertility: a review of the literature. Fertil Steril. 2001;75(2):237-48.

13.Sloter E, Schmid TE, Marchetti F, Eskenazi B, Nath $\mathrm{J}$, Wyrobek AJ. Quantitative effects of male age on sperm motion. Hum Reprod. 2006;21(11):2868-75.

14.Pasqualotto FF, Sobreiro BP, Hallak J, Pasqualotto EB, Lucon AM. Cigarette smoking is related to a decrease in semen volume in a population of fertile men. BJU Int. 2006;97(2):324-6.

15.Sepaniak S, Forges T, Gerard H, Foliguet B, Bene MC, Monnier-Barbarino P. The influence of cigarette smoking on human sperm quality and
DNA fragmentation. Toxicology. 2006;223(1-2): 54-60.

16.Queiroz EK, Waissmann W. Occupational exposure and effects on the male reproductive system. Cad Saúde Pública. 2006;22(3):485-93.

17. Pasqualotto FF, Lucon AM, Sobreiro BP, Pasqualotto EB, Arap S. Effects of medical therapy, alcohol, smoking and endocrine disruptors on male infertility. Rev Hosp Clin Fac Med São Paulo. 2004;59(6):375-82.

18.World Health Organization. Laboratory manual for the examination of human semen and sperm-cervical mucus interaction. Cambridge: Cambridge University Press; 1999.

19. Menkveld R, Stander F, Kotze T, Kruger T, Zyl JV. The evaluation of morphological characteristics of human spermatozoa according to strict criteria. Hum Reprod. 1990;5(5):586-92.

20.Gorelick JI, Goldstein M. Loss of fertility in man with varicocele. Fertil Steril. 1993;59(3):613-6.

21.Witt MA, Lipshultz LI. Varicocele: a progressive or static lesion? Urology. 1993;42(5):541-3.

22.Vine MF. Smoking and male reproduction: a review. Int J Androl. 1996;19(6):323-37.

23.Vogt HJ, Heller WD, Borelli S. Sperm quality of healthy smokers, ex-smokers, and never smokers. Fertil Steril. 1986; 45(1):106-10.

24.Zinaman MJ, Brown CC, Selevan SG, Clegg ED. Semen quality and human fertility: a prospective study with healthy couples. J Androl. 2000;21(1):145-53.

25. Kuhnert B, Nieschlag E. Reproductive functions of the ageing male. Hum Reprod Update. 2004;10(4):327-39. 